\title{
Influence of aging and gadolinium exposure on T1, T2, and T2*-relaxation in healthy women with an increased risk of breast cancer with and without prior exposure to gadoterate meglumine at 3.0-T brain MR imaging
}

\author{
Kathrin Barbara Krug ${ }^{1}$ (D) $\cdot$ Christina Jane Burke ${ }^{1} \cdot$ Kilian Weiss $^{1,2} \cdot$ Pascal A. T. Baltzer $^{3} \cdot$ Kerstin Rhiem $^{4}$. \\ David Maintz ${ }^{1} \cdot$ Marc Schlamann $^{1} \cdot$ Martin Hellmich $^{5}$
}

Received: 15 February 2021 / Revised: 30 April 2021 / Accepted: 11 May 2021 / Published online: 3 July 2021

(C) The Author(s) 2021

\begin{abstract}
Objectives We examined the effects of aging and of gadolinium-based contrast agent (GBCA) exposure on MRI measurements in brain nuclei of healthy women.

Methods This prospective, IRB-approved single-center case-control study enrolled 100 healthy participants of our high-risk screening center for hereditary breast cancer, who had received at least six doses of macrocyclic GBCA (exposed group) or were newly entering the program (GBCA-naïve group). The cutoff "at least six doses" was chosen to be able to include a sufficient number of highly exposed participants. All participants underwent unenhanced 3.0-T brain MRI including quantitative T1, T2, and R2* mapping and T1- and T2-weighted imaging. The relaxation times/signal intensities were derived from region of interest measurements in the brain nuclei performed by a radiologist and a neuroradiologist, both board certified. Statistical analysis was based on descriptive evaluations and uni-/multivariable analyses.

Results The participants (exposed group: 49, control group: 51) were aged $42 \pm 9$ years. In a multivariable model, age had a clear impact on R2* $(p<0.001-0.012)$, T2 $(p=0.003-0.048)$, and T1 relaxation times/signal intensities $(p<0.004-0.046)$ for the majority of deep brain nuclei, mostly affecting the substantia nigra, globus pallidus (GP), nucleus ruber, thalamus, and dentate nucleus (DN). The effect of prior GBCA administration on T1 relaxation times was statistically significant for the DN, GP, and pons $(p=0.019$ 0.037).

Conclusions In a homogeneous group of young to middle-aged healthy females aging had an effect on T2 and R2* relaxation times and former GBCA applications influenced the measured T1 relaxation times.

Key Points

- $\quad$ The quantitative $T 1, T 2$, and $R 2 *$ relaxation times measured in women at high risk of developing breast cancer showed characteristic bandwidth for all brain nuclei examined at 3.0-T MRI.

- The effect of participant age had a comparatively strong impact on $R 2$ *, T2, and T1 relaxation times for the majority of brain nuclei examined.

- The effect of prior GBCA administrations on T1 relaxation times rates was comparatively less pronounced, yielding statistically significant results for the dentate nucleus, globus pallidus, and pons.
\end{abstract}

Kathrin Barbara Krug

Barbara.krug@uk-koeln.de

1 Department of Diagnostic and Interventional Radiology, University Hospital of Cologne, Kerpener Straße 62, 50937 Cologne, Germany

2 Philips Medical Systems, Hamburg, Germany

3 Department of Biomedical Imaging and Image-guided Therapy, Division of General Radiology, Allgemeines Krankenhaus, Medical University of Vienna, Vienna, Austria
4 Center for Hereditary Breast and Ovarian Cancer, Center for Integrated Oncology (CIO), Faculty of Medicine and University Hospital of Cologne, University of Cologne, Cologne, Germany

5 Institute of Medical Statistics and Bioinformatics, University of Cologne, Cologne, Germany 
Summary statement Healthy women with and without previous GBCA-enhanced breast MRI exhibited age-related T2* and T2 relaxation alterations at 3.0 T-brain MRI. T1 relaxation alterations due to prior GBCA administration were comparatively less pronounced.

Keywords Gadolinium $\cdot$ Contrast media $\cdot$ Middle aged $\cdot$ Globus pallidus $\cdot$ Cerebellar nuclei

$\begin{array}{ll}\text { Abbreviations } \\ \text { CA } & \text { Crus anterior of the internal capsule } \\ \text { CN } & \text { Caudate nucleus } \\ \text { DN } & \text { Dentate nucleus } \\ \text { GBCA } & \text { Gadolinium-based contrast agents } \\ \text { Gd } & \text { Gadolinium } \\ \text { GP } & \text { Globus pallidus } \\ \text { LL } & \text { Look-Locker } \\ \text { NR } & \text { Nucleus ruber } \\ \text { PO } & \text { Pons } \\ \text { PU } & \text { Putamen } \\ \text { SN } & \text { Substantia nigra } \\ \text { TH } & \text { Thalamus } \\ \text { VFA } & \text { Variable flip-angle }\end{array}$

\section{Introduction}

Although relaxation changes due to aging and ingestion of metalliferous foods are widely considered to be present in healthy adults, there is a paucity of data on typical visible changes on MR imaging [1-4]. Contrary to the majority of chemical elements normally ingested, the earth metal gadolinium $(\mathrm{Gd})$ is not a physiologically inherent component of the human body [5-7]. Since their clinical introduction in 1988, gadolinium-based contrast agents (GBCAs) were considered to have an excellent safety profile with reported serious adverse reactions in the range of $0.03 \%$ [6, 8]. Since 2014, however, retrospective studies have indicated an association between previous GBCA administration and increased signal intensity, predominantly in the dentate nucleus and the globus pallidus, on unenhanced T1-weighted MR images $[9,10]$. The observed signal intensity changes seemed dose dependent and to be associated more often with linear GBCAs than macrocyclic GBCAs in rodent and human studies [6, 9, 11-20]. Harmful side effects of $\mathrm{Gd}$ deposits in the brain have been discussed [21]. However, a recent population-based study found no association between prior Gd exposure and neurodegenerative disease [22]. Although the long-term clinical relevance of cerebral Gd-deposits currently remains unknown [7], for safety reasons, the use of linear GBCAs has been prohibited in the European Union since 2017/2018 [7, 23].

Due to their high paramagnetic properties, GBCAs are of pivotal importance for contrast-enhanced breast MRI which constitutes the most sensitive method for early breast cancer detection available [24-28]. Therefore, women with an increased genetic risk of developing breast cancer aged 30 to 50 years undergo annual GBCA-enhanced breast MR imaging like in most international intensified screening programs $[26$, 27]. This group thus represents a unique collective of healthy women inherently at risk of Gd exposure from GBCA administration.

Recently, a case-control study reported no GBCAassociated T1 signal increases in brain nuclei exposed to $\geq 6$ GBCA doses and unexposed controls [29]. The study was limited by age differences between cases and controls and examined $\mathrm{T} 1$ relaxation only. The current investigation aims to extend this preliminary evidence and thus was based on a larger number of MRI examinations and a balanced casecontrol population regarding participants' age. We pursued the working hypothesis:

- That it is possible to generate quantitative T1, T2, and $\mathrm{R} 2 *(1 / \mathrm{T} 2 *)$ acquisition sequences with a spatial and contrast resolution suitable for detecting GBCA- and ageassociated relaxation time differences in brain nuclei and

- That we succeed in demonstrating possible age- and dosedependent relaxation time changes and signal intensity changes in brain nuclei following multiple intravenous doses of GBCA compared to Gd-naïve controls.

\section{Material and methods}

\section{Study participants}

This single-center, investigator-initiated, prospective casecontrol study was carried out with the approval of the institutional ethics review board (file reference 16-240) and was not supported by industrial sponsoring.

All women included were attenders of the local center of the National Intensified Early Breast Cancer Detection Program. They were at high risk of developing breast cancer, had no history of cancer or neurological disease, and had provided written informed consent to take part in the study. The women in the exposed group had previously received at least six doses of macrocyclic GBCA in the context of surveillance. The threshold of 6 GBCA-guided breast MRI examinations was chosen as trade-off between the increasing 


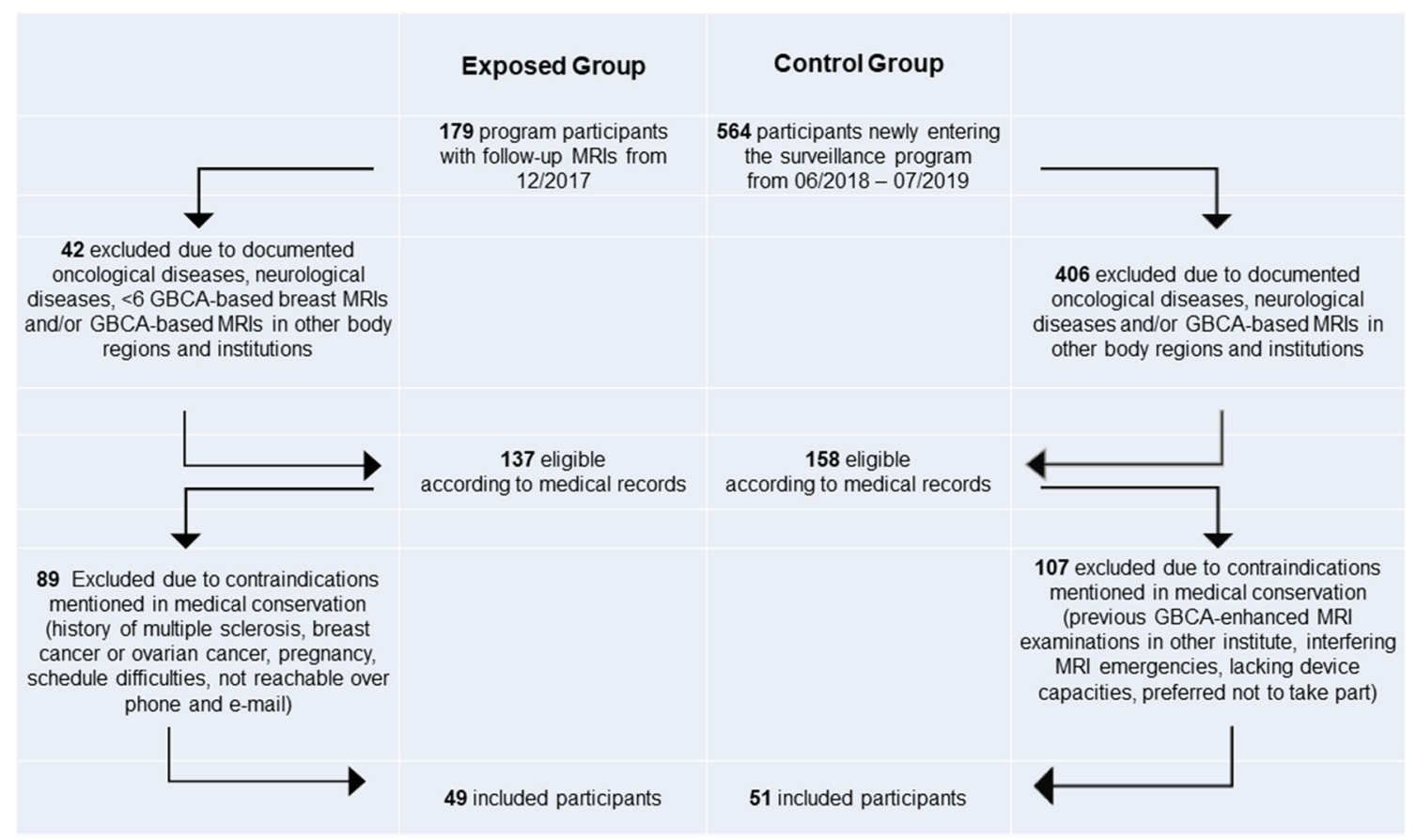

Fig. 1 Recruitment of the participants taking part in the exposed group and in the control group

likelihood of Gd retention with higher numbers of GBCA exposures and the number of healthy advice-seekers at our family breast center with higher numbers of GBCA applications. The control group comprised women newly entering the program with no prior GBCA exposure and were prospectively included during a period of 11 months. All women eligible for study participation were identified via the comprehensive electronic Hospital and Radiology Information Systems of the University Hospital (ORBIS ${ }^{\circledR}$ OpenMed/RIS Nice ${ }^{\circledR}$, Dedalus Healthcare Systems Group) (Fig. 1). Inclusion and exclusion criteria were confirmed via telephone calls and onsite consultations (B.K.). The members of the exposed group had never received any other contrast medium other than gadoterate meglumine and the controls had never received any GBCA according to their own account and all available medical data. Eligible participants with uncertain medical history, a history of cancer or neurological disease, compromised kidney function, and/or $<6$ contrast-based breast MRIs, contrast-based MRIs of other body regions (exposed group), or any GBCA-enhanced MRIs (controls) were excluded.

The breast MRI protocols used were in accordance with international recommendations [30]. Gadoterate meglumine (Dotarem ${ }^{\circledR}$, Guerbet $\mathrm{GmbH}$ ) was the only contrast agent used at our institution since 2006. The contrast medium was injected via a cubital vein at a dose of $0.1 \mathrm{mmol} / \mathrm{kg}$ body weight. The total number and cumulative volume of GBCA administered were obtained from the electronic Radiology Information System.

\section{MR imaging}

All participants underwent unenhanced MR imaging of the brain using a clinical 3.0-T scanner (Ingenia 3, Philips Healthcare) and a vendor-supplied 16-channel head coil. The median time interval between the last GBCA-based breast MRI and the native brain MRI examination was 89 days (range 18 to 254 days). The participants were positioned head first in supine position in the MRI scanner. Image data acquisition was in axial plane. It included T1 mapping for the quantification of $\mathrm{T} 1$ relaxation using a 2D Inversion Recovery Look-Locker (LL) sequence and a B1-corrected 3D Variable Flip-Angle (VFA) Gradient Echo sequence, T2 mapping for the quantification of T2 relaxation using a 2D Multi Gradient Spin Echo sequence, and R2* mapping for R2* quantification using a 2D Multi Gradient Echo sequence (Table 1). Furthermore, T1-weighted 2D Spin Echo (T1wSEM), T1weighted 3D Turbo Gradient Echo (T1w3D), and T2weighted 2D Turbo Spin Echo sequences (T2wTSE) were carried out in order to obtain T1- and T2-weighted images.

\section{Image analysis}

Image data were pseudonymized and stored as uncompressed DICOM files in PACS (ImpaxEE®, Dedalus Healthcare Systems Group). Image data were analyzed using a dedicated PACS-workstation. The region of interest (ROI) was initially placed by one investigator 


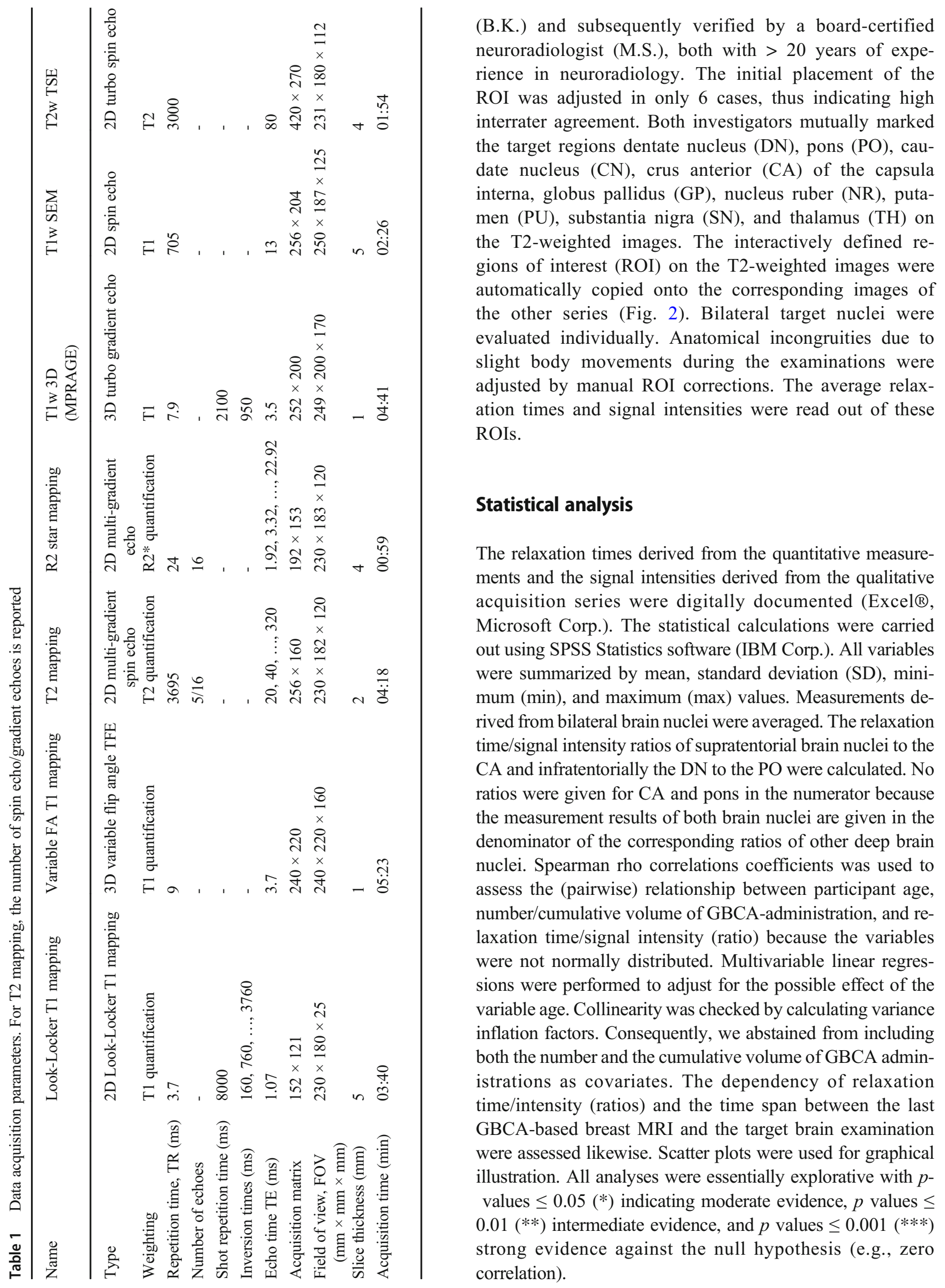


Fig. 2 Anatomy-based placement of regions-of-interests (ROI) in a 48-year-old study participant who had received 13 doses of gadolinium-based contrast agents (GBCA) with a cumulative volume of $194 \mathrm{~mL}$ in the last 9 years prior to the target brain MRI. a: ROI placements in the dentate nucleus (a), the pons (b), the substantia nigra and the nucleus ruber (c), and in the crus anterior of the internal capsule (d) in the T2wTSE acquisition sequence. b: ROI placements in the globus palidus, the putamen, the caudate nucleus, and the thalamus for the acquisition sequences Look-Locker (LL) T1 mapping (a), variable flip angle (VFA) T1 mapping (b), T2 mapping (c), and R2* mapping (d). c: ROI placements in the globus palidus, the putamen, the caudate nucleus, and the thalamus for the qualitative T1w3D sequence (a), the qualitative T1wSEM (b), and the qualitative T2wTSE sequence
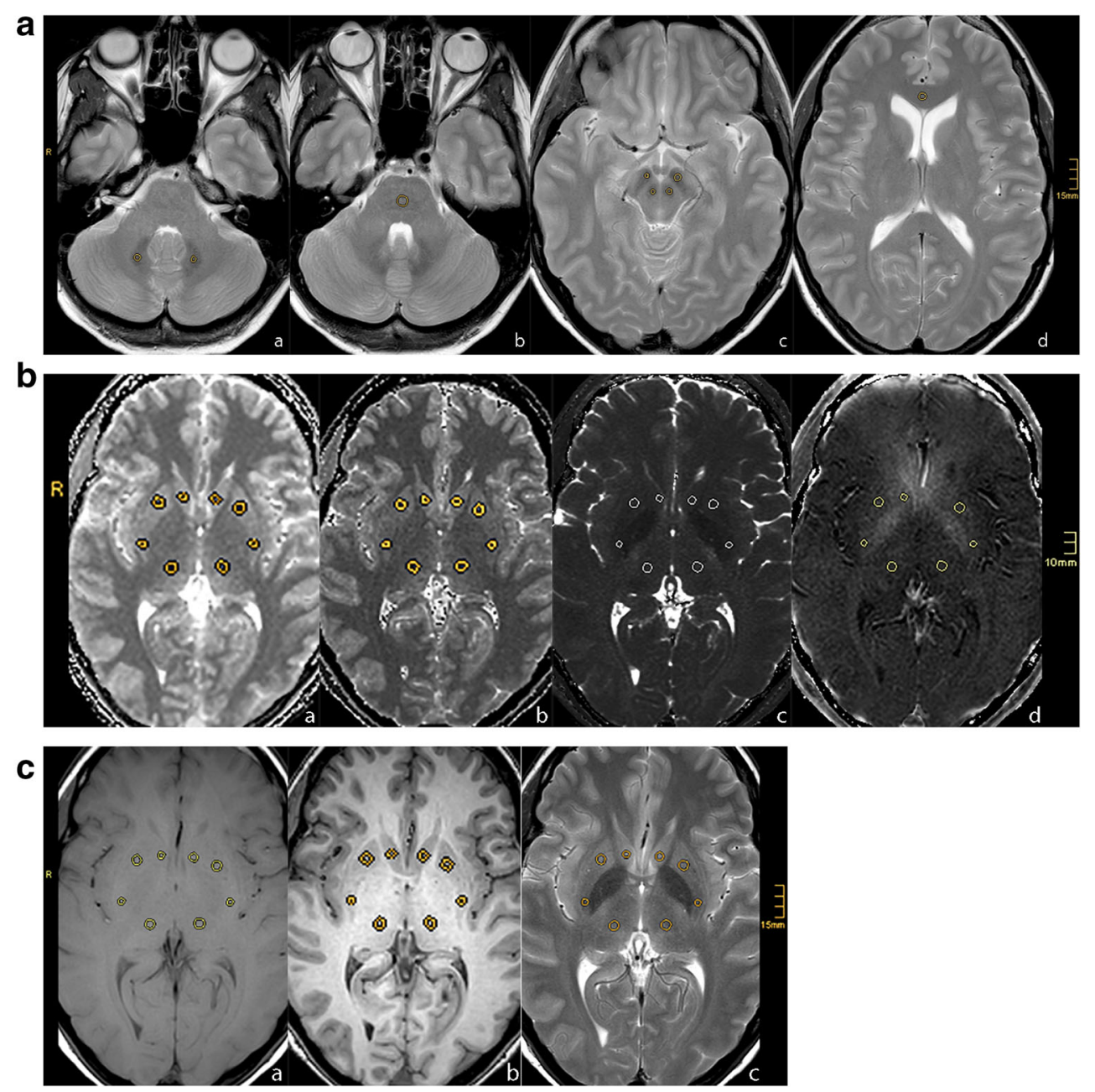

\section{Results}

\section{Study participants}

The study group consisted of 100 women with a median age of 41.5 years (minimum 24 years, maximum 63 years) (Table 2). The age distribution did not differ between both groups (exposed: $42(24-63)$ years, controls: $38(28-57)$ years). The exposed participants had received a median of 8.0 doses (range: 6 to 14 doses) of gadoterate meglumine prior to the target brain MRI, the median cumulative GBCA dose being $119 \mathrm{~mL}$ ( 69 to $194 \mathrm{~mL}$ ). The mean time interval between the last GBCA-enhanced breast MRI and the target brain MRI was $104 \pm 55$ days (18 to 254 days). Diagnostic assessment of the acquired brain MRI images yielded normal results in all study examinations.
Table 2 Participant

demographics. All participants were female. Data are given as numbers (n), mean \pm standard deviation or median and range. $G B C A$ gadolinium-based contrast agent, $y$ years

\begin{tabular}{ll}
\hline Parameter & Value \\
\hline Total no. of participants & 100 \\
Mean age (y) & $41.5(24-63)$ \\
Participants without GBCA exposure & 51 \\
Mean age (y) & $42(24-63)$ \\
Participants exposed to GBCA & 49 \\
Mean age (y) & $38(28-57)$ \\
Mean time interval since last GBCA exposure (days) & $104 \pm 55$ \\
Minimum and maximum time interval since last GBCA exposure (days) & $18 ; 254$ \\
Median cumulative gadoterate meglumine doses (mL) & $119(69-194)$ \\
Median number of gadoterate meglumine doses & $8.0(6-14)$ \\
\hline
\end{tabular}




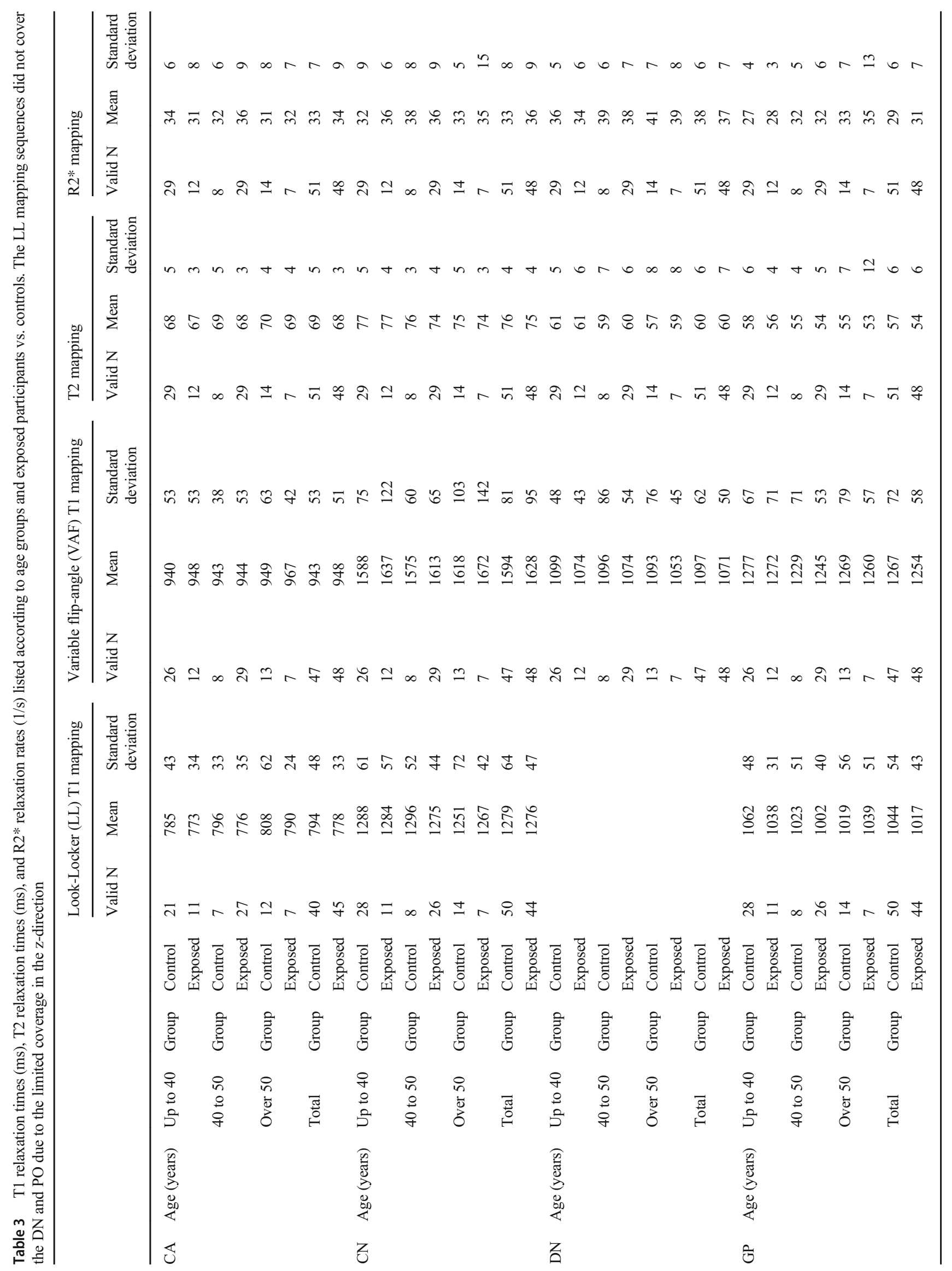




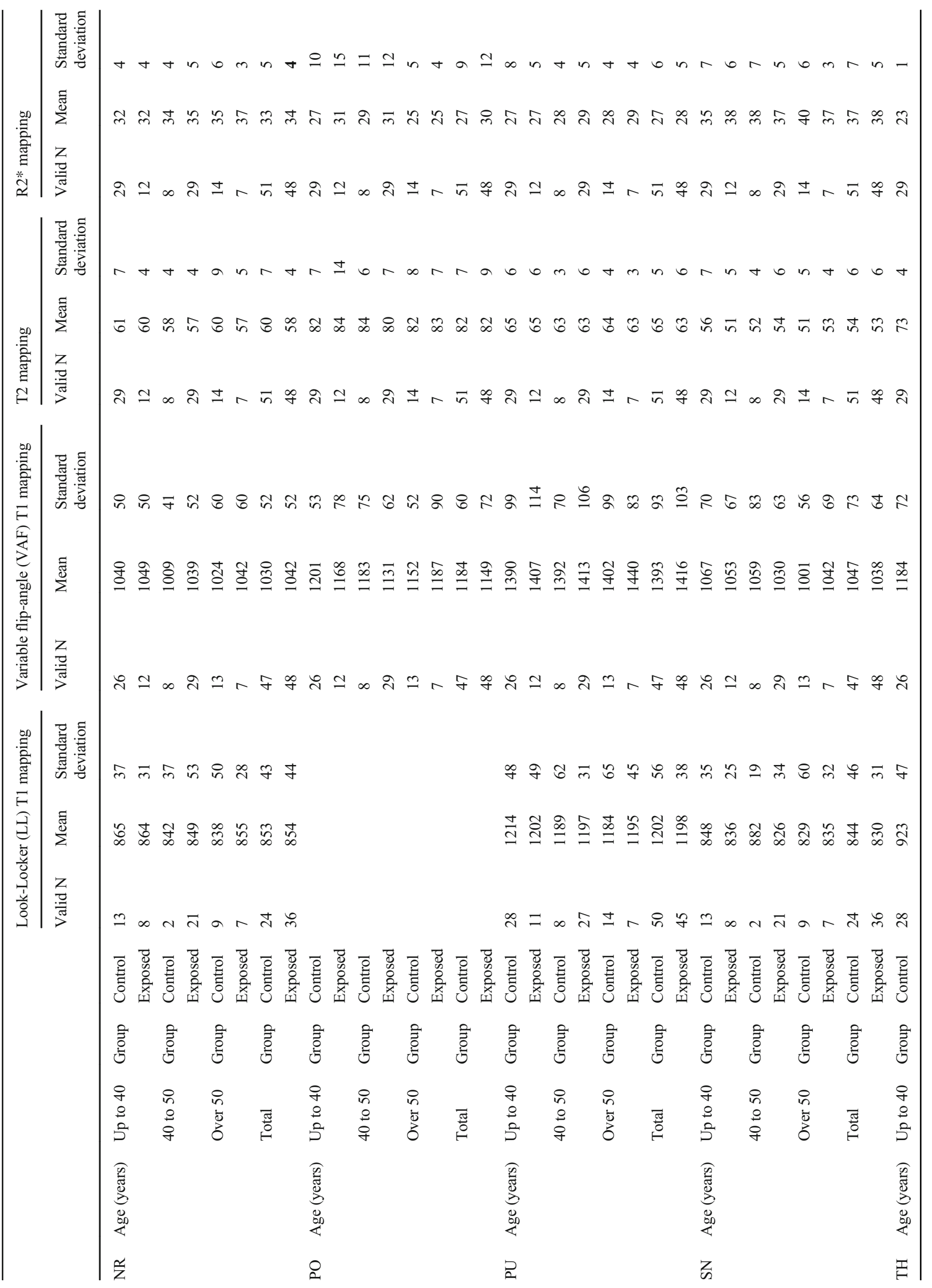




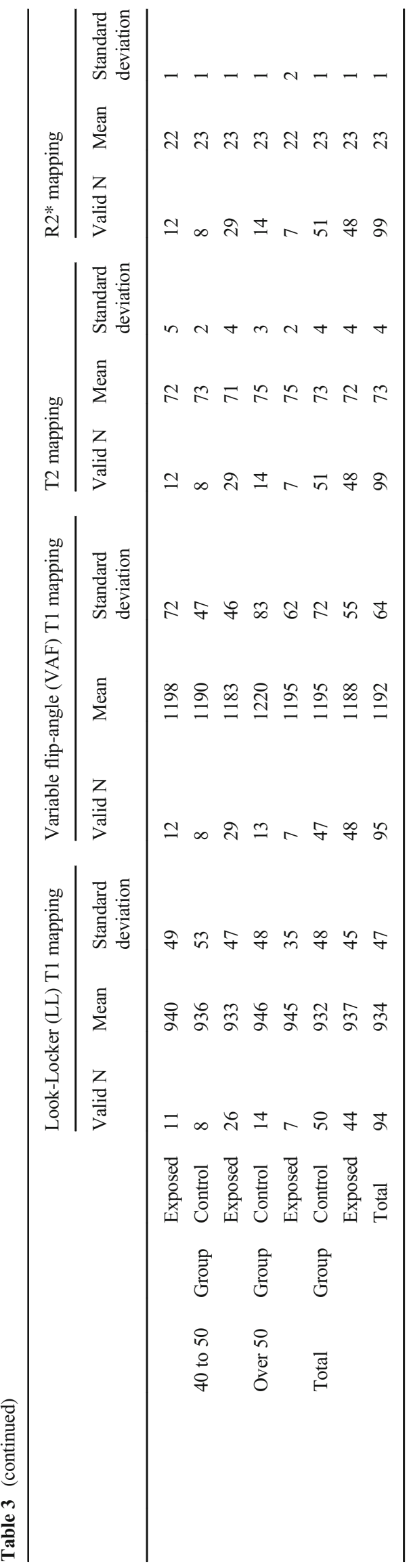

\section{Quantitative MRI data acquisitions}

\section{Relaxation times}

The numeric differences between the participants exposed to $\geq$ 6 GBCA doses and the GBCA naïve controls were indiscernible on the MR images.

The quantitative relaxation times for all examined brain regions showed homogeneous distributions for all acquisition sequences in the examined brain nuclei (Table 3 ). The T1 relaxation times measured by LL mapping were consistently higher than those of VFA mapping and differed systematically in the same direction (range: $\triangle \mathrm{CA} 161 \mathrm{~ms}$ to $\triangle \mathrm{CN} 333 \mathrm{~ms}$ ). The shortest T1 relaxation was measured in the CA (794 \pm 48 $\mathrm{ms} / 948 \pm 51 \mathrm{~ms})$ and the longest in the CN $(1279 \pm 64 \mathrm{~ms} /$ $16281 \pm 95 \mathrm{~ms})$.

\section{Univariable comparisons}

The effect variable "age" revealed negative correlation coefficients with increasing age for T1 mapping in both groups and for the majority of brain nuclei reaching moderate significance in the controls in the GP $(p=0.025)$, PO $(p=0.025)$, and SN ( $p=0.013)$ (Table 4, Fig. 3a). The correlation coefficients for "age" were also mostly negative in T2 mapping reaching moderate to high significance in the GP and the SN (controls: $p=0.047$ and 0.006) and NR (exposed: $p=0.039$ ). Due to the inverse read out compared to the $\mathrm{T} 2 *$ relaxation times, the R2* correlation coefficients were mostly positive reaching moderate to high significance in the control group (DN $p<0.000, \mathrm{NR} p=0.020$, GP $p<0.000$, SN $p<0.021$ ) and in the exposed group (DN $p=0.009, \mathrm{NR} p=0.033$ ).

The time interval between the last GBCA-based breast MRI and the target brain MRI (effect variable "decay") negatively correlated with $\mathrm{T} 1$ relaxation times measured in most brain nuclei reaching moderate significance in the CA and GP ( $p=0.032$ and 0.040 ). The correlation of the effect variable "decay" and measured R2* relaxation values was mostly positive due to the sign inversion compared to T2* times showing moderate significance in the DN and GP ( $p=0.040$ and 0.03$)$.

The collinear effect variables "number" and "cumulative volume" of applied GBCA doses correlated negatively with most of the measured $\mathrm{T} 1$ relaxation times without reaching statistical significance due to the shortening of $\mathrm{T} 1$ relaxation caused by GBCA. The T2 measurements showed indecisive results, whereas the correlation coefficients in $\mathrm{R} 2 *$ mapping were mostly positive without reaching statistical significance.

\section{Multivariable analyses}

Multivariable analysis of the effect variables "age" and "group comparison," the latter defined as the comparison of the GBCA-exposed group and the GBCA-naïve controls, 


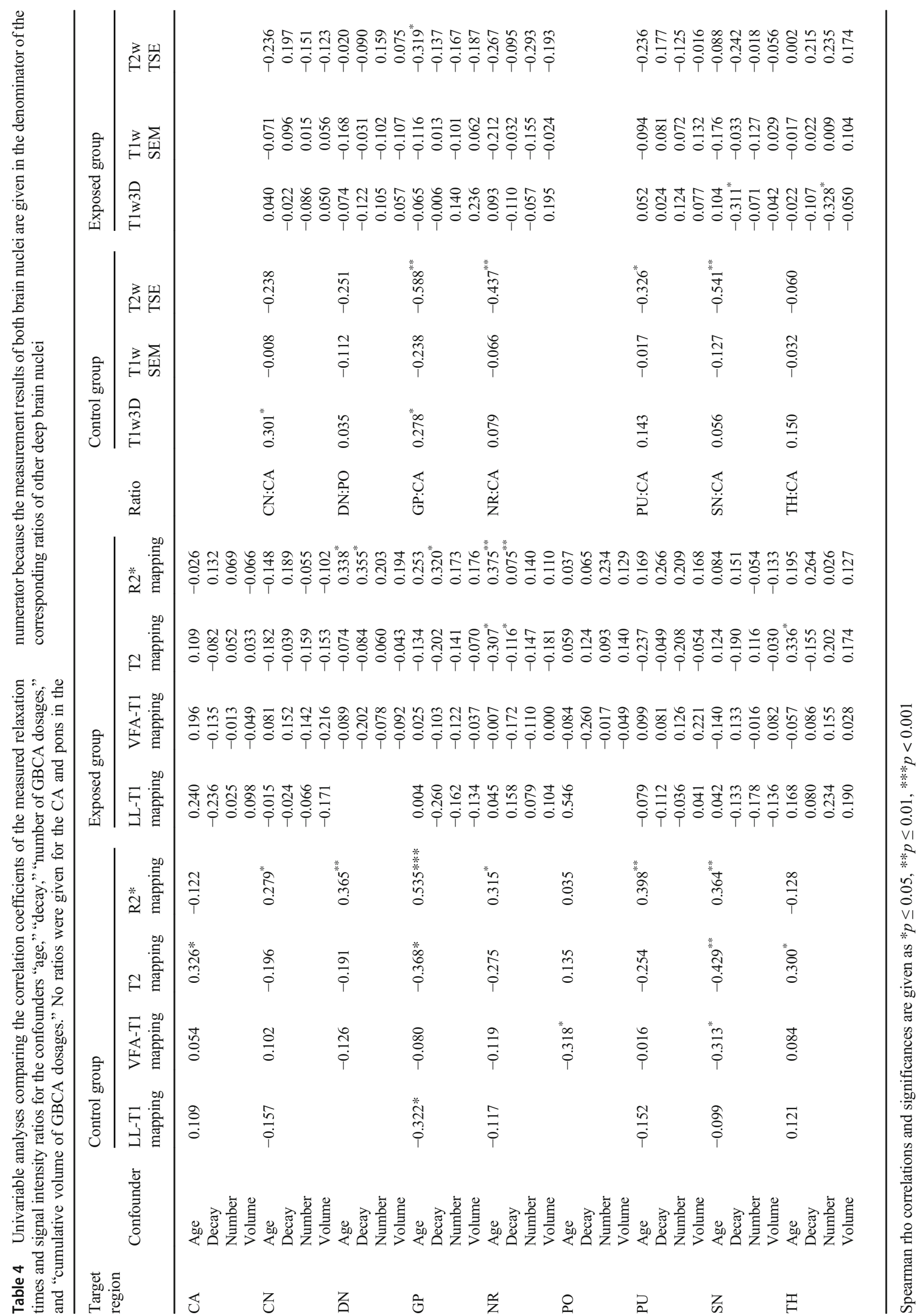


a
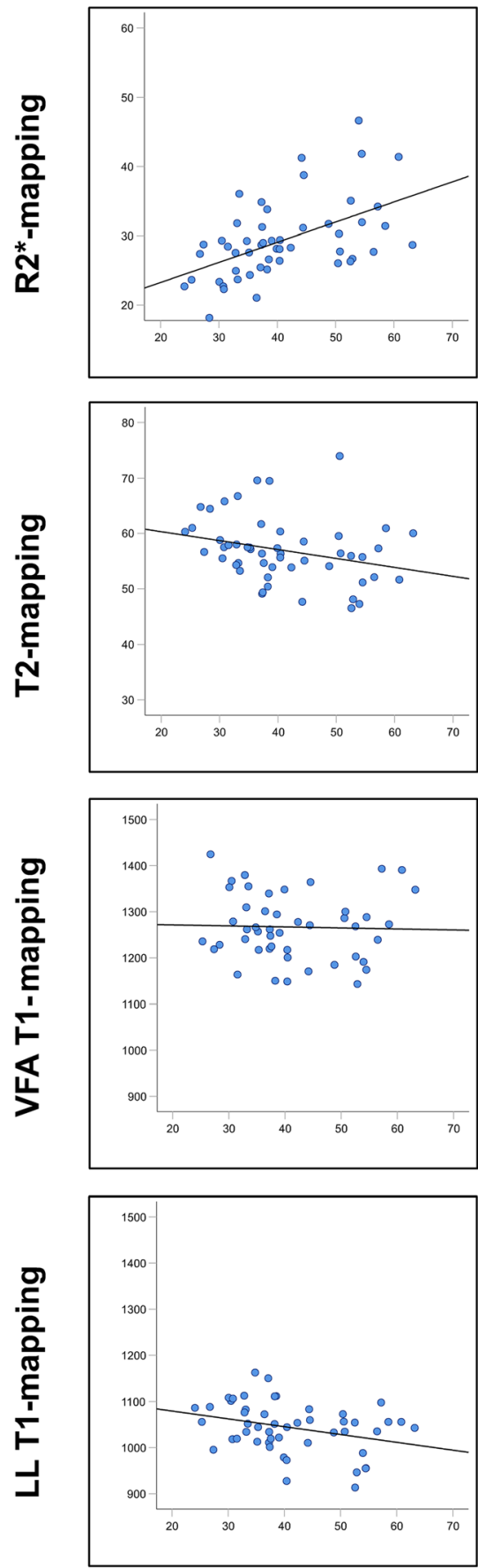

Age (years)
EXPOSED
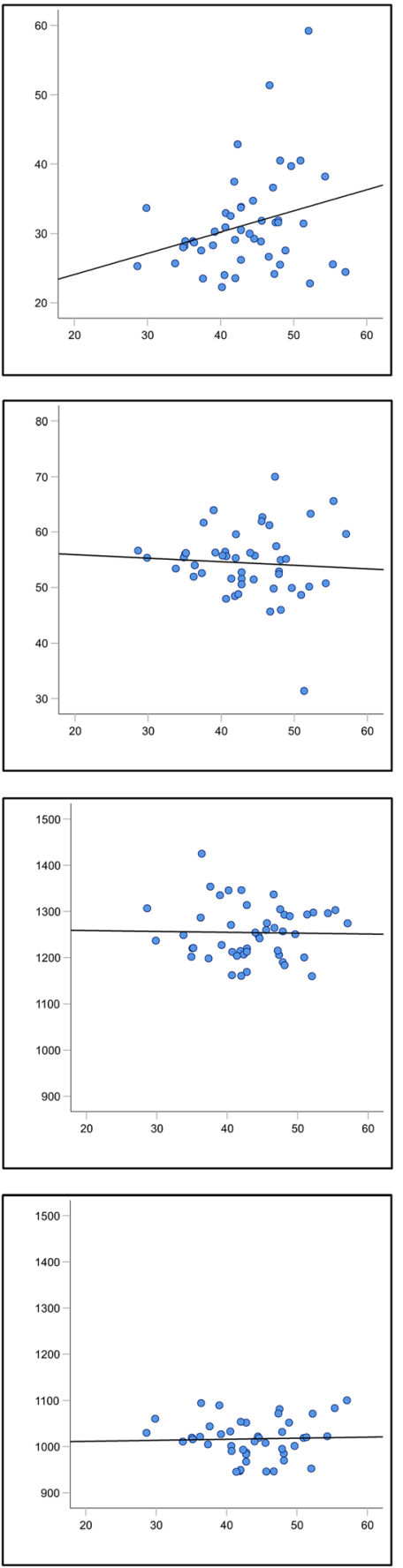

Age (years)
EXPOSED
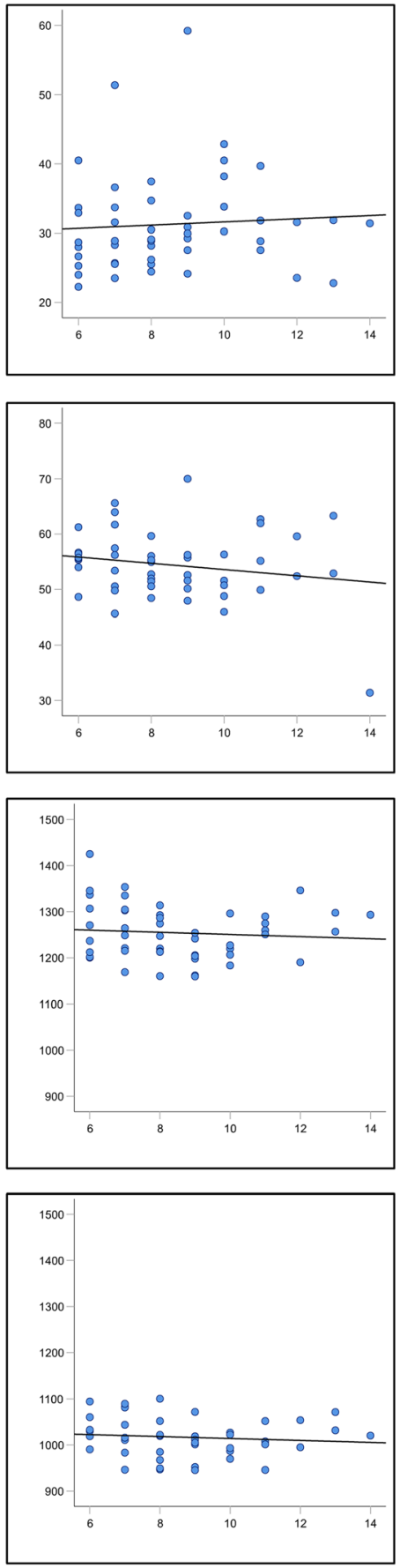

Number of GBCA applications
Fig. 3 Interdependency of age (left and middle columns) and the number of prior GBCA doses (right column) and the relaxation time(s)/signal intensity ratios measured in the globus pallidus (GP) and the crus anterior (CA) of the internal capsule. a: R2* mapping, T2 mapping,
VAFT1 mapping, and LL-T1 mapping in the GP. b: Ratios GP:CA for R2* mapping, T2 mapping, VFA 1 mapping and LL-T1 mapping. c: Ratios GP:CA for the qualitative T1-weighted and T2-weighted data acquisition sequences confirmed significant correlations for "age" in the GP ( $p=$ $0.005)$ and $\mathrm{SN}(p=0.046)$ in T1 mapping and moderate significance in the CA ( $p=0.038), \mathrm{CN}(p=0.024), \mathrm{NR}(p=$ $0.048), \mathrm{SN}(p=0.048)$, and TH $(p=0.015)$ in T2 mapping 
b CONTROL
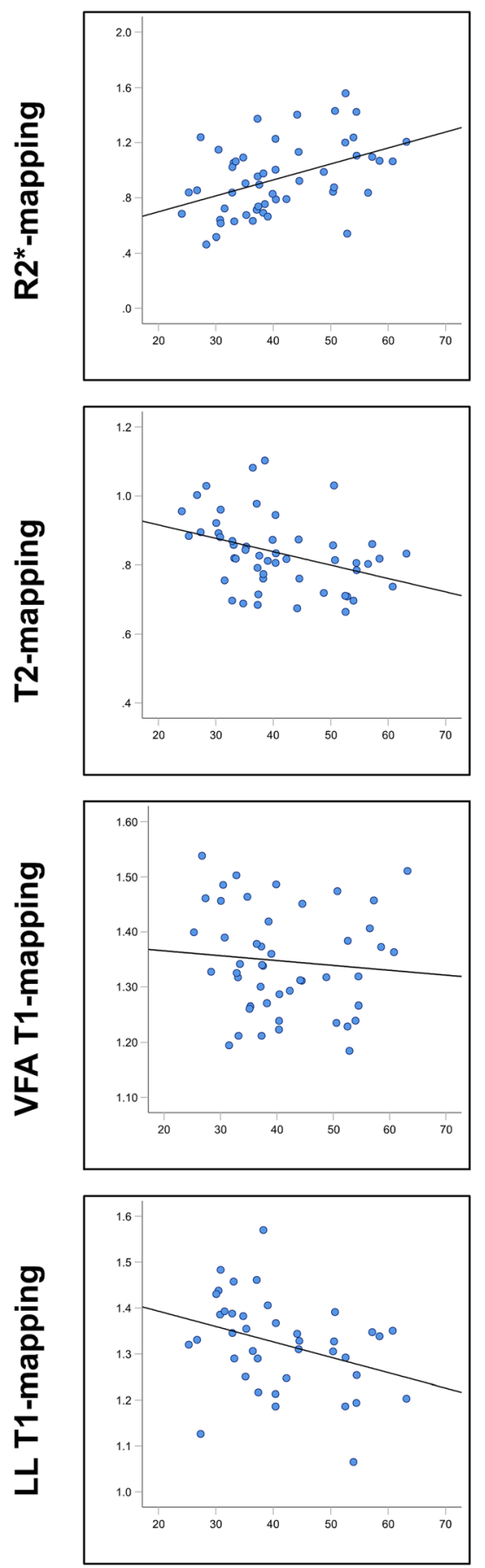

Age (years)
EXPOSED
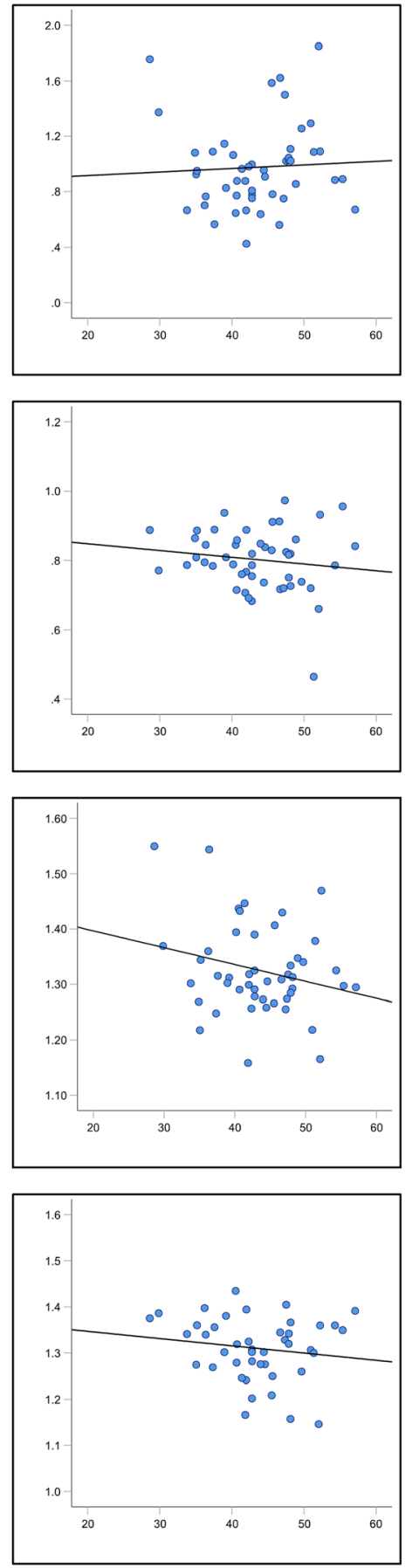

Age (years)
EXPOSED
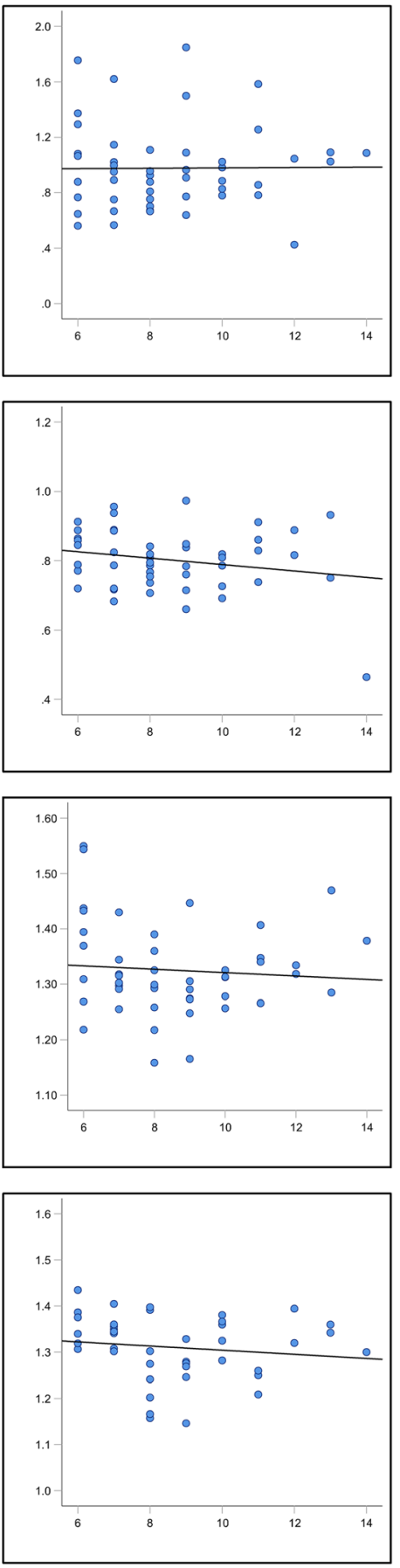

Number of GBCA applications

Fig. 3 (continued)

(Table E1). "Age" had a highly significant influence on the relaxation rates in $\mathrm{R} 2 *$ mapping in the $\mathrm{DN}(p=0.001), \mathrm{GP}(p$ $<0.000)$, NR $(p=0.001)$, and SN $(p=0.02)$.
Regarding the effect variable "group comparison," which reflects the overall difference between the exposed group and the control group and not just the impact of GBCA, 
C
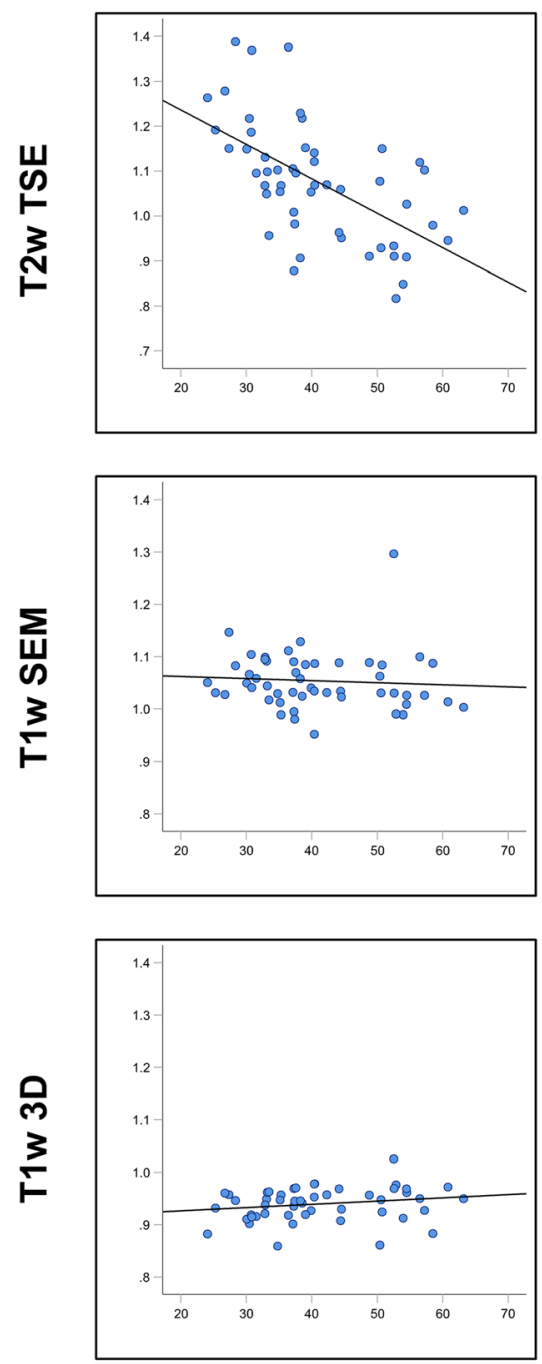

Age (years)
EXPOSED
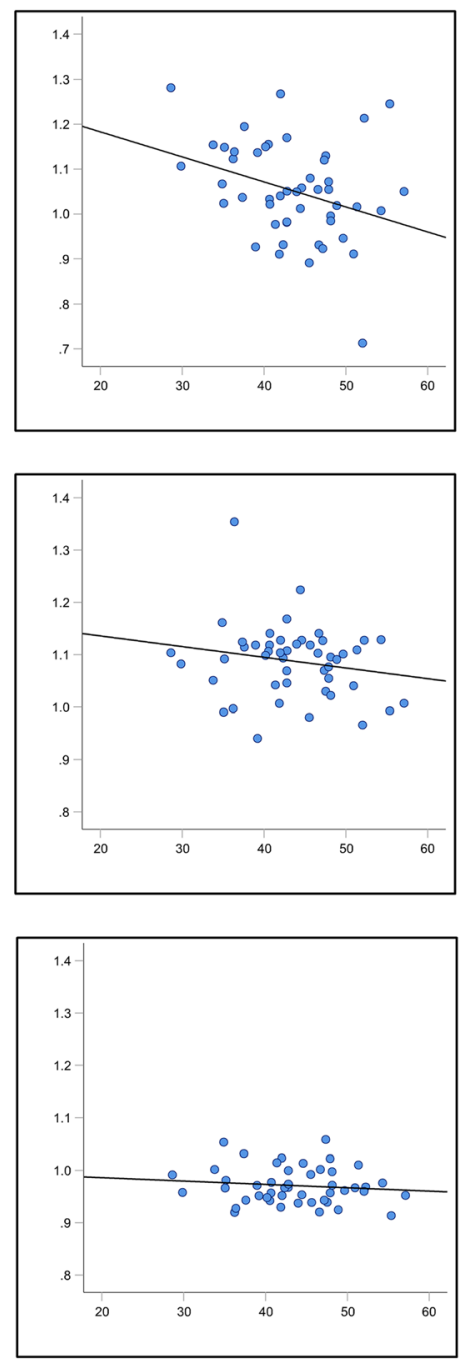

Age (years)
EXPOSED
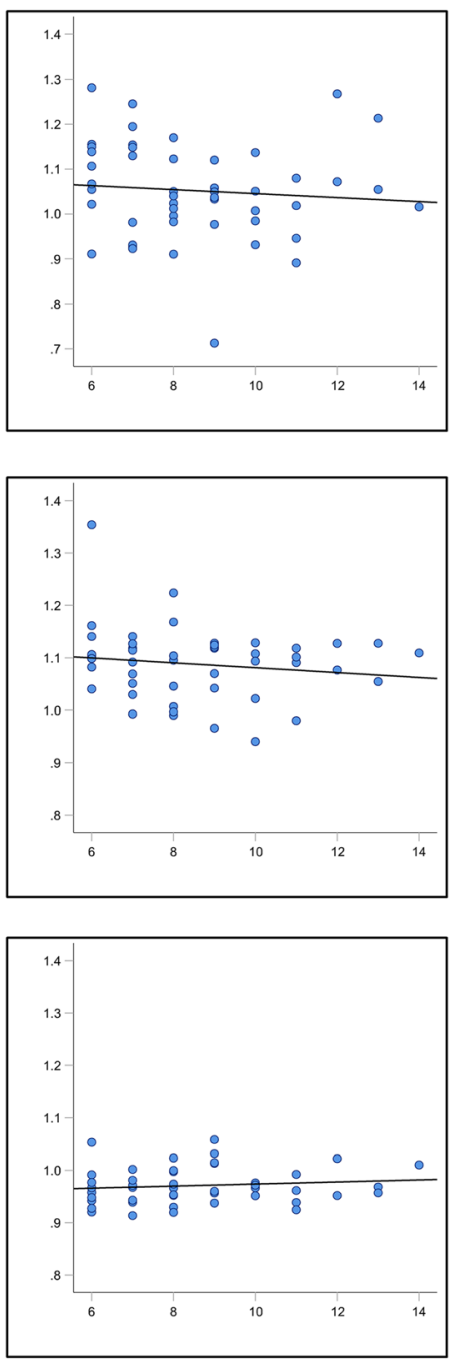

Number of GBCA applications

Fig. 3 (continued)

multivariable analysis revealed negative orientations of the slope coefficients (B-values) correlating with a tendency towards a shortening of T1 relaxation times due to GBCA application. The observed negative correlations were moderately significant in the CA $(p=0.037)$ in LL mapping and in the DN $(p=0.033)$ and $\mathrm{PO}(p=0.019)$ in VFA mapping. We did not notice any significant effect of the effect variable "group comparison" regarding $\mathrm{T} 2$ and $\mathrm{R} 2 *$ mapping.

These results were confirmed for "age" when normalizing the relaxation times for each participant by calculating intraindividual ratios between the relaxation times measured in the target brain nuclei and in the CA (supratentorial) and $\mathrm{PO}$ (infratentorial) (Table E1, Fig. 3b). The calculated slope coefficients were predominantly negative in $\mathrm{T} 1 / \mathrm{T} 2$ mapping and positive in $\mathrm{R} 2 *$ mapping due to the inverse read out of the $\mathrm{T} 2 *$ signal. The influence of "age" on the relaxation time ratios showed moderate to intermediate significance for ratios $\mathrm{SN}: \mathrm{CA}(p=0.004), \mathrm{CN}: \mathrm{CA}(p=0.021), \mathrm{NR}: \mathrm{CA}(p=$ $0.013)$, GP:CA $(p=0.010)$, and GP:TH $(p=0.031)$ in LL mapping, for the ratio $\mathrm{CN}$ :CA in in VFA mapping ( $p=$ $0.005)$, for the ratio $\mathrm{PU}: \mathrm{CA}(p=0.022), \mathrm{CN}: \mathrm{CA}(p=$ $0.003), \mathrm{NR}: \mathrm{CA}(p=0.003), \mathrm{GP}: \mathrm{CA}(p=0.004), \mathrm{GP}: \mathrm{TH}(p$ $=0.002)$, and $\mathrm{SN}: \mathrm{CA}(p=0.007)$ in T2 mapping and moderate to strong correlations for ratios DN:CA $(p=0.018), \mathrm{GP}: \mathrm{CA}(p$ $=0.007)$, and GP:TH $(p<0.001)$ in R2* mapping.

The negative slope coefficients observed for the "number of GBCA applications" in the CA $(p=0.036), \mathrm{DN}(p=0.040)$, GP $(p=0.024)$, and PO $(p=0.026)$ (Table E2) and for the collinear effective variable "cumulative volume of applied GBCA doses" in the GP $(p=0.024)$ and $\mathrm{PO}(p=0.026)$ reflect 
a shortening of $\mathrm{T} 1$ times with increasing numbers of applied GBCA doses per exposed study participant (Table E3). Likewise, the ratios of the relaxation times showed negative B-values in T1/T2 mapping and positive B-values in R2* mapping. The variable "age" had a moderate to intermediate influence on the ratios CN:CA $(p=0.019), \mathrm{NR}: \mathrm{PO}(p=$ $0.007)$, GP:CA $(p=0.012)$, and SN:CA $(p=0.003)$ in LL mapping and an intermediate level of significance for the ratio SN:CA $(p=0.006)$ in VFA mapping when regarding the number of applied GBCA doses. Correspondingly, the assessment of the effect variables "age" and "cumulative volume" of applied GBCA doses yielded correlations of moderate to intermediate significance for the ratios $\mathrm{CN}$ :CA $(p=0.020)$, NR:PO $(p=0.008)$, GP:CA $(p=0.013)$, GP:TH $(p=$ $0.047)$, and SN:CA $(p=0.003)$. The influence of the effect variable "age" on the collinear effect variables "number" and "cumulative volume" of applied GBCA doses reached moderate significance for PU:CA $(p=0.025$ and 0.022), CN:CA ( $p=0.003$ and 0.004), NR:PO $(p=0.004$ and 0.005), GP:CA $(p=0.006$ and 0.006), GP:TH $(p=0.003$ and 0.003$)$, and SN:CA $(p=0.005$ and 0.006 ) in T2 mapping and moderate to strong significance for DN:PO ( $p=0.010$ and 0.012), GP:CA $(p=0.007$ and 0.008), and GP:TH $(p<0.000$ and $<0.000)$ in R2* mapping (Tables E2 and E3).

\section{Qualitative MRI data acquisitions}

Univariable and multivariable analyses of the T1- and T2weighted qualitative acquisition sequences for signal intensity essentially confirmed the aforementioned quantitative results (Tables 4 and Table E1). Multivariable analysis of the effect variables "age" and "group comparison" confirmed a significant correlation between "age" and the T2-weighted signal intensity ratios $\mathrm{CN}$ :CA $(p=0.003), \mathrm{NR}: \mathrm{CA}(p<0.001), \mathrm{GP}: \mathrm{CA}$ $(p<0.001)$, GP:Th $(p<0.001), \mathrm{PU}: \mathrm{CA}(p<0.001)$, and SN:CA $(p<0.001)$ (Table E1, Fig. 3c). In both T1-weighted sequences, the effect variable "group comparison" revealed moderate to strong correlations for the ratios $\mathrm{CN}$ :CA $(p=0.031$ and 0.004), GP:CA ( $p<0.001$ and 0.005), and SN:CA $(p=0.010$ and 0.046$)$. The number and cumulative volume of applied GBCA doses showed similar results (Tables E2 and E3).

\section{Discussion}

Our observation that the effect of aging on relaxation times measured in brain nuclei of young to middle-aged healthy females was predominantly seen as an age-related decrease in $\mathrm{T} 2 *$ relaxation time $(\mathrm{DN}, \mathrm{GP}, \mathrm{NR}: p \leq 0.001)$ and in $\mathrm{T} 2$ relaxation time $(\mathrm{CN}, \mathrm{NR}, \mathrm{SN}: p=0.015-0.048)$ and an only moderate decrease in T1 relaxation time for the GP $(p=0.046)$ is in accordance with preclinical studies, which have shown age-related concentration changes of iron, copper, and zinc in brain nuclei as well as associated microglial and astrocyte alterations [1-4]. Contrary to iron, copper, and zinc, the earth metal Gd is not a physiologically inherent component of the human body [5-7]. Because of its toxicity, Gd must be bound to a ligand in order to render it safe to be administered intravenously while maintaining its paramagnetic properties for MRI [7, 30]. Although it is known that a small fraction of intravenously applied GBCA is chronically retained in human tissues [14], little is known about its biochemical transformation, handling, and excretion [6, 7]. Histological studies have shown evidence of Gd deposition in brain tissue of patients with normal renal function [14, 17, 18, 31]. Spheroid Gd deposits were demonstrated in the basal lamina of cerebral microvessels and in the perivascular Virchow-Robin spaces of rodents after repeated injections of linear GBCAs [32]. The mechanism for deposition has not yet been fully elucidated and the clinical significance remains unclear [31, 33, 34].

Our results regarding quantitative T1 mapping with a 3.0-T MRI system are in accordance with the findings of Saake et al who, using a 1.5-T scanner, investigated 160 patients with multiple GBCA administrations and 60 GBCA-naïve control subjects and found significantly shortened $\mathrm{T} 1$ relaxation times in the GP in the exposed group [34]. Our findings also confirm those of Quattrocchi et al [24], who assessed the effect of age and number of previous injections of linear GBCA on signal intensity of unenhanced T1-weighted images of the DN and GP in 892 patients with prior GBCA exposure and 1906 subjects without. The results revealed a correlation of the signal intensity ratios DN:PO and GP:TH with age and the number of macrocyclic GBCA injections.

In the current study, quantitative T1/T2 mapping and qualitative T1/T2-weighted imaging were employed in order to investigate the effects of aging and Gd deposits on relaxation time. While quantitative relaxation values provide absolute measures and thus should allow for direct interindividual comparisons, they also imply the challenge of careful interpretation due to technical dependencies, which may have an impact on the quantification of results. Two different sequence designs were used for quantitative T1 mapping. The systematically higher relaxation time values in VFA mapping are explained by the differences in both sequence design, regarding spatial resolution and contrast resolution, and the number of $\mathrm{T} 1$ relaxation points assessed. T1 relaxation was read out at five points in LL mapping, whereas only two T1 relaxation read outs were carried out during the VFA acquisitions in order to gain a sufficient compromise between spatial resolution, contrast resolution, and the length of examination time. The presented ratio approach for the qualitative and quantitative measurements was chosen in order to ameliorate the influence of interindividual variances due to the individual concentrations of physiologically ingested metal ions like copper and iron in the brain nuclei examined, which cannot be 
quantified from patient history like the case with prior GBCA dosing.

The main limitation of the presented study lies in the interindividual comparison of healthy young women of the same age group. The lack of an intraindividual approach will be addressed in an ongoing study including attenders of the screening program. Further limitations may be seen in the small number of cases and the monocentric study design, as well as in the yet informal evaluation of interrater agreement on the basis of the ROI placements.

In conclusion, the known effects of aging and $\mathrm{Gd}$ exposure could be observed in the brain nuclei of a clinically homogeneous group of female adults using quantitative and qualitative 3.0-T MR imaging. The effect of aging was most pronounced in $\mathrm{T} 2$ and $\mathrm{T} 2 * \mathrm{MR}$ imaging. The influence of prior GBCA applications on the measured T1 relaxation times and T1-weighted signal intensities were too small to be visually perceived on the MR images of brain nuclei.

Supplementary Information The online version contains supplementary material available at https://doi.org/10.1007/s00330-021-08069-4.

Funding Open Access funding enabled and organized by Projekt DEAL.

\section{Declarations}

Guarantor The scientific guarantor of this publication is Prof. Dr. Med. Kathrin Barbara Krug.

Conflict of interest The authors of this manuscript declare no relationships with any companies whose products or services may be related to the subject matter of the article.

Statistics and biometry Prof. Martin Hellmich, one of the authors of the manuscript, kindly provided statistical advice for this manuscript and carried out the biometric evaluations.

Informed consent Written informed consent was obtained from all subjects (patients) in this study.

Ethical approval Institutional Review Board approval was obtained.

\section{Methodology \\ - Prospective \\ - Diagnostic or prognostic study \\ - Performed at one institution}

Open Access This article is licensed under a Creative Commons Attribution 4.0 International License, which permits use, sharing, adaptation, distribution and reproduction in any medium or format, as long as you give appropriate credit to the original author(s) and the source, provide a link to the Creative Commons licence, and indicate if changes were made. The images or other third party material in this article are included in the article's Creative Commons licence, unless indicated otherwise in a credit line to the material. If material is not included in the article's Creative Commons licence and your intended use is not permitted by statutory regulation or exceeds the permitted use, you will need to obtain permission directly from the copyright holder. To view a copy of this licence, visit http://creativecommons.org/licenses/by/4.0/.

\section{References}

1. Ashraf A, Michaelides C, Walker TA (2019) Regional distributions of iron, copper and zinc and their relationships with glia in a normal aging mouse model. Front Aging Neurosci 11:351. https://doi.org/ 10.3389/fnagi.2019.00351

2. Erikson KM, Syversen T, Steinnes E, Aschner M (2004) Globus pallidus: a target brain region for divalent metal accumulation associated with dietary iron deficiency. J Nutr Biochem 15:335-341

3. Martin-Bastida A, Pietracupa S, Piccini P (2017) Neuromelanin in parkinsonian disorders: an update. Int J Neurosci 127:1116-1123

4. Zecca L, Youdim MB, Riederer P, Connor JR, Crichton RR (2004) Iron, brain ageing and neurodegenerative disorders. Nat Rev Neurosci 5:863-873

5. Behra-Miellet J, Gressier B, Brunet C et al (1996) Free gadolinium and gadodiamide, a gadolinium chelate used in magnetic resonance imaging: evaluation of their in vitro effects on human neutrophil viability. Methods Find Exp Clin Pharmacol 18:437-442

6. McDonald RJ, Levine D, Weinreb J et al (2018) Gadolinium retention: a research roadmap from the $2018 \mathrm{NIH} / \mathrm{ACR} / \mathrm{RSNA}$ workshop on gadolinium chelates. Radiology 289:517-534

7. Runge VM (2018) Dechelation (Transmetalation): Consequences and safety concerns with the linear gadolinium-based contrast agents, in view of recent health care rulings by the EMA (Europe), FDA (United States), and PMDA (Japan). Invest Radiol 53:571-578

8. Costello JR, Kalb B, Martin DR (2016) Incidence and risk factors for gadolinium-based contrast agent immediate reactions. Top Magn Reson Imaging 25(6):257-263

9. Kanda T, Ishii K, Kawaguchi H, Kitajima K, Takenaka D (2014) High signal intensity in the dentate nucleus and globus pallidus on unenhanced T1-weighted MR images: relationship with increasing cumulative dose of a gadolinium-based contrast material. Radiology 270:834-841

10. Radbruch A, Weberling LD, Kieslich PJ et al (2015) Gadolinium retention in the dentate nucleus and globus pallidus is dependent on the class of contrast agent. Radiology. 275:783-791

11. Frenzel T, Apte C, Jost G, Schöckel L, Lohrke J, Pietsch H (2017) Quantification and assessment of the chemical form of residual gadolinium in the brain after repeated administration of gadolinium-based contrast agents: comparative study in rats. Invest Radiol 52:396-404

12. Jost G, Lenhard DC, Sieber MA, Lohrke J, Frenzel T, Pietsch H (2016) Signal increase on unenhanced T1-weighted images in the rat brain after repeated, extended doses of gadolinium-based contrast agents: comparison of linear and macrocyclic agents. Invest Radiol 51:83-89

13. Murata N, Gonzalez-Cuyar LF, Murata K et al (2016) Macrocyclic and other non-group 1 gadolinium contrast agents deposit low levels of gadolinium in brain and bone tissue: preliminary results from 9 patients with normal renal function. Invest Radiol 51:447453

14. Bjørnerud A, Vatnehol SAS, Larsson C, Due-Tønnessen P, Hol PK, Groote IR (2017) Signal enhancement of the dentate nucleus at unenhanced MR imaging after very high cumulative doses of the macrocyclic gadolinium-based contrast agent gadobutrol: an observational study. Radiology 285:434-444

15. Cao Y, Huang DQ, Shih G, Prince MR (2016) Signal change in the dentate nucleus on T1-weighted MR images after multiple 
administrations of gadopentetate dimeglumine versus gadobutrol. AJR Am J Roentgenol 206:414-419

16. Kang KM, Choi SH, Hwang M, Yun TJ, Kim J, Sohn C-H (2017) T1 Shortening in the Globus Pallidus after multiple administrations of gadobutrol: assessment with a multidynamic multiecho sequence. Radiology 287:258-266

17. McDonald RJ, McDonald JS, Kallmes DF et al (2015) Intracranial Gadolinium deposition after contrast-enhanced MR imaging. Radiology 275:772-782

18. McDonald RJ, McDonald JS, Kallmes DF et al (2017) Gadolinium deposition in human brain tissues after contrast-enhanced MR imaging in adult patients without intracranial abnormalities. Radiology 285:546-554

19. Radbruch A, Haase R, Kickingereder P et al (2017) Pediatric Brain: No increased signal intensity in the dentate nucleus on unenhanced T1-weighted MR images after consecutive exposure to a macrocyclic gadolinium-based contrast agent. Radiology 283:828-836

20. Quattrocchi CC, Ramalho J, van der Molen AJ, Rovira À, Radbruch A, GREC, European Gadolinium Retention Evaluation Consortium and the ESNR, European Society of Neuroradiology (2019) Standardized assessment of the signal intensity increase on unenhanced T1-weighted images in the brain: the European Gadolinium Retention Evaluation Consortium (GREC) Task Force position statement. Eur Radiol 29:3959-3967

21. Semelka RC, Ramalho J, Vakharia A et al (2016) Gadolinium deposition disease: initial description of a disease that has been around for a while. Magn Reson Imaging 34:1383-1390

22. Welk B, McArthur E, Morrow SA et al (2016) Association between gadolinium contrast exposure and the risk of Parkinsonism. JAMA 316:96-98

23. European Medicines Agency (EMA) (2017) EMA's final opinion confirms restrictions on use of linear gadolinium agents in body scans EMA/625317/2017 Nov p. 4Report No.: EMA/625317/ 2017. http://www.ema.europa.eu/docs/en_GB/document_library/ Referrals_document/gadolinium_contrast_agents_31/European Commission final decision/W $\overline{\mathrm{W}} 5002 \overline{4} 0575 . \overline{\mathrm{pdf}}$. Accesse $\overline{\mathrm{d}}$ February 20, 2020

24. Quattrocchi CC, Errante Y, Mallio CA et al (2018) Effect of age on high T1 signal intensity of the dentate nucleus and globus pallidus in a large population exposed to gadodiamide. Invest Radiol 53: 214-222
25. Bennani-Baiti B, Bennani-Baiti N, Baltzer PA (2016) Diagnostic performance of breast Magnetic Resonance Imaging in noncalcified equivocal breast findings: results from a systematic review and meta-analysis. PLoS One 11(8):e0160346. https://doi.org/10. 1371/journal.pone. 0160346

26. Bick U, Engel C, Krug B et al (2019) High-risk breast cancer surveillance with MRI: 10-year experience from the German Consortium for Hereditary Breast and Ovarian Cancer. Breast Cancer Res Treat 175:217-228

27. Lee CS, Monticciolo DL, Moy L (2020) Screening Guidelines Update for Average-Risk and High-Risk Women. AJR Am J Roentgenol 214:316-323

28. Mann RM, Cho N, Moy L (2019) Breast MRI: State of the Art. Radiology. 292:520-536

29. Bennani-Baiti B, Krug B, Giese D et al (2019) Evaluation of 3.0-T MRI brain signal after exposure to gadoterate meglumine in women with high breast cancer risk and screening breast MRI. Radiology 293:523-530

30. Mann RM, Kuhl CK, Kinkel K, Boetes C (2008) Breast MRI: guidelines from the European Society of Breast Imaging. Eur Radiol 18:130-1318

31. Taoka T, Naganawa S (2018) Gadolinium-based contrast media, cerebrospinal fluid and the glymphatic system: possible mechanisms for the deposition of gadolinium in the brain. Magn Reson Med Sci 17:111-119

32. Hao D, Ai T, Goerner H, Runge VM, Tweedle M (2012) MRI contrast agents: basic chemistry and safety. J Magn Reson Imaging 36:1060-1071

33. Rasschaert M, Schroeder JA, Wu T-D et al (2018) Multimodal imaging study of gadolinium presence in rat cerebellum: differences between Gd chelates, presence in the Virchow-Robin space, association with lipofuscin, and hypotheses about distribution pathway. Invest Radiol 53:518-528

34. Saake M, Schmidle A, Kopp M et al (2019) MRI brain signal intensity and relaxation times in individuals with prior exposure to gadobutrol. Radiology. 290:659-668

Publisher's note Springer Nature remains neutral with regard to jurisdictional claims in published maps and institutional affiliations. 